\title{
Erratum to: Expression of myeloperoxidase and gene mutations in AML patients with normal karyotype: double $C E B P A$ mutations are associated with high percentage of MPO positivity in leukemic blasts
}

\author{
Shinya Tominaga-Sato $\cdot$ Hideki Tsushima $\cdot$ Koji Ando $\cdot$ Hidehiro Itonaga $\cdot$ \\ Yoshitaka Imaizumi · Daisuke Imanishi $\cdot$ Masako Iwanaga $\cdot$ Jun Taguchi · \\ Takuya Fukushima $\cdot$ Shinichiro Yoshida $\cdot$ Tomoko Hata $\cdot$ Yukiyoshi Moriuchi • \\ Kazutaka Kuriyama $\cdot$ Hiroyuki Mano $\cdot$ Masao Tomonaga $\cdot$ Yasushi Miyazaki
}

Published online: 20 July 2012

(C) The Japanese Society of Hematology 2012

Erratum to: Int J Hematol (2011) 94:81-89

DOI 10.1007/s12185-011-0883-y

The original version of this article unfortunately contained some errors in Table 2 in the column headed "Amino acid changes". The corrected table is given here.

We sincerely apologize for the errors.

The online version of the original article can be found under doi:10.1007/s12185-011-0883-y.

S. Tominaga-Sato $\cdot$ H. Itonaga $\cdot$ M. Iwanaga $\cdot$ J. Taguchi

Y. Miyazaki

Department of Hematology and Molecular Medicine Unit, Atomic Bomb Disease Institute,

Nagasaki University Graduate School of Biomedical Sciences,

Nagasaki, Nagasaki, Japan

H. Tsushima $(\bowtie) \cdot$ Y. Imaizumi · D. Imanishi ·

T. Fukushima $\cdot$ T. Hata

Department of Hematology,

Nagasaki University Hospital,

1-7-1 Sakamoto, Nagasaki 852-8501, Japan

e-mail: tsushima@nagasaki-u.ac.jp

K. Ando $\cdot$ S. Yoshida

Department of Internal Medicine,

Nagasaki National Medical Center,

Ohmura, Nagasaki, Japan

Y. Moriuchi

Division of Hematology,

Sasebo City General Hospital,

Sasebo, Nagasaki, Japan

K. Kuriyama

School of Health Sciences, University of the Ryukyus,

Okinawa, Nishihara, Japan

H. Mano

Division of Functional Genomics,

Jichi Medical University, Shimotsuke, Tochigi, Japan

M. Tomonaga

Department of Hematology,

Japanese Red-Cross Nagasaki Atomic Bomb Hospital,

Nagasaki, Nagasaki, Japan 


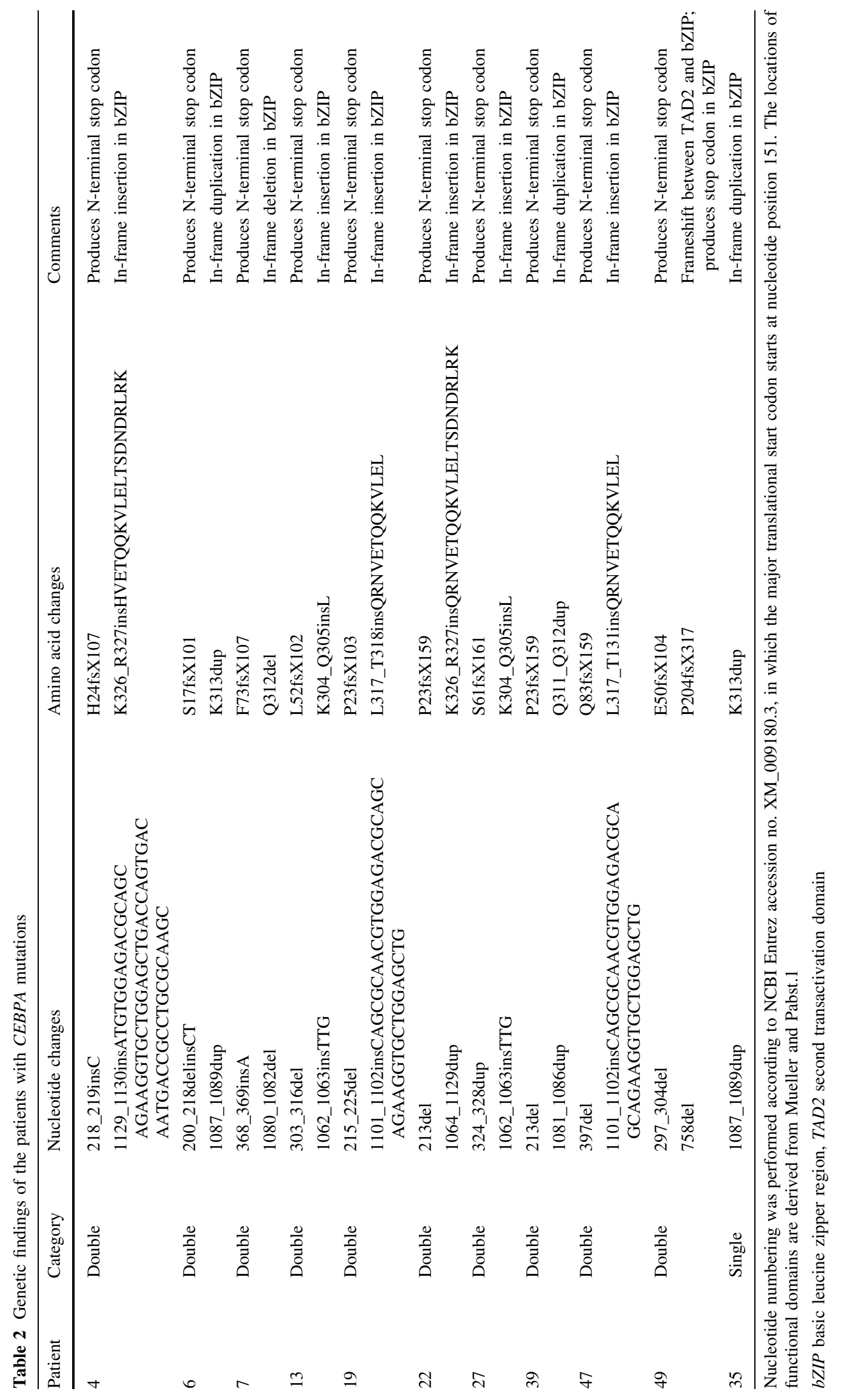

